\title{
O uso de canabinoides em fisiopatologias dermatológicas: uma revisão sistemática
}

The use of cannabinoids in dermatological pathophysiologies: a systematic review

El uso de cannabinoides en fisiopatología dermatológica: una revisión sistemática

Maria Gabryelle da Silva Soares ORCID: https://orcid.org/0000-0003-2575-8267 Universidade Federal de Campina Grande, Brasil E-mail: gabryelle8685@gmail.com

Maria Eduarda Wanderley de Barros Silva ORCID: https://orcid.org/0000-0002-4642-3282 Universidade Federal de Campina Grande, Brasil

E-mail: eduarda.wanderley@ outlook.com

Maria Letícia Cardoso da Silva Barbosa ORCID: https://orcid.org/0000-0002-2935-6882 Universidade Federal de Campina Grande, Brasil

E-mail: marialeticia20151@ @otmail.com

Lidinês Pereira de Macêdo

ORCID: https://orcid.org/0000-0001-8421-6527 Universidade Federal de Campina Grande, Brasil E-mail: lindinezpereira09@gmail.com

Francisco Jacinto Silva Santos Júnior ORCID: https://orcid.org/0000-0002-3457-7057 Universidade Potiguar, Brasi E-mail: juni0rs4nt00@gmail.com

Allan César Avelino Costa ORCID: https://orcid.org/0000-0001-8390-538X Universidade Potiguar, Brasi E-mail: allancesar.costaa@gmail.com

Alexandre Henrique Silva de Mâcedo ORCID: https://orcid.org/0000-0002-0526-4588 Universidade Potiguar, Brasil E-mail: alexhenriquesm@gmail.com

Àlex Espinalt Daví Lima de Freitas ORCID: https://orcid.org/0000-0001-7820-8969 Universidade Potiguar, Brasil

E-mail: alexespinalt.lima@gmail.com

Gabriel Fragoso Peixoto

ORCID: https://orcid.org/0000-0003-0640-6602 Universidade Tiradentes, Brasil

E-mail: gabriel.fpeixoto@souunit.com.br

Vitória Liz de Souza Correia ORCID: https://orcid.org/0000-0001-8277-1623 Universidade Tiradentes, Brasil E-mail: vitoriali@hotmail.com

Ana Paula Moura Manzini ORCID: https://orcid.org/0000-0003-1864-7024 Universidade Tiradentes, Brasil E-mail: apmmanzini@gmail.com

Maria Eduarda de Lima Onório ORCID: https://orcid.org/0000-0002-4701-2282 Escola de Ensino Superior do Agreste Paraibano, Brasil

E-mail: m.eduardaonorio.enf@gmail.com

Rita de Cássia Rodrigues Lopes ORCID: https://orcid.org/0000-0002-7596-1629 Escola de Ensino Superior do Agreste Paraibano, Brasil

E-mail: ritadecassiarodrigueslopes@ hotmail.com

Yngrid Lima Pedreira

ORCID: https://orcid.org/0000-0003-4135-1125

Universidade Salvador, Brasil

E-mail: yngrid.pedreira@gmail.com

Larissa Lima Soares

ORCID: https://orcid.org/0000-0002-7682-372X Centro Universitário Tiradentes, Brasil E-mail: larissalyma@hotmail.com 


\title{
Resumo
}

O referido artigo trata-se de uma revisão bibliográfica sistemática realizada no período de 25 de outubro a 04 de novembro 2021 através de pesquisa eletrônica nas bases de dados do Google Acadêmico, Science Direct e PUBMEDNCBI-NIH para obtenção de dados mais recentes, a questão norteadora da pesquisa foi: Qual a importância dos canabinoides para o tratamento de fisiopatologias dermatológicas? Como critério de elegibilidade foi selecionado artigos dos últimos três anos referentes à cannabis, seus canabinoides e o tratamento de lesões dermatológicas. Os descritores utilizados foram: Cannabis, Skin Diseases e Cannabinoids verificados nos Descritores em Ciência da Saúde (DeCS) e no Medical Subject Headings (MeSH). Sucedeu-se o cruzamento utilizando- se do operador booleano AND: "Cannabis" AND "Skindiseases" AND "Cannabinoids". Foram eleitos 9 artigos, ambos analisados de forma qualitativa, através de leitura metodológica. Mesmo que a erva medicinal seja conhecida por seu uso psicológico, estudos recentes mostram que os canabinóides (CBs), estão ativamente envolvidos em sistemas periféricos, como a resposta imune, inata e adaptativa. O sistema endocanabinóide é responsável e funcional por regular diversos aspectos da homeostasia da pele como proliferação, liberação e diferenciação de mediadores inflamatórios. Portanto, foi possível observar através da pesquisa que a cannabis sativa e seu sistema presente no organismo, tem grandes participações para o controle de diversas doenças dermatológicas e não dermatológicas, contudo, estudos ainda são bastante recentes e escassos a respeito do efeito da erva na área dermatológica, no entanto, já tem grandes avanços sobre sua eficácia.

Palavras-chave: Cannabis; Canabinoides; Dermatopatias.

\begin{abstract}
This article is a systematic bibliographic review carried out in the period from October 25 to November 4, 2021 through electronic search in the databases of Google Scholar, Science Direct and PUBMED-NCBI-NIH to obtain more recent data, the guiding question of the research was: What is the importance of cannabinoids for the treatment of dermatological physiopathology? The eligibility criteria were selected articles from the last three years concerning cannabis, its cannabinoids and the treatment of dermatological lesions. The descriptors used were: Cannabis, Skin diseases and Cannabinoids verified in Health Science Descriptors (Decs) and Medical Subjec the adings (Mesh). The cross was succeeded using the boolean operator AND: "Cannabis" AND "Skindiseases" AND "Cannabinoids". Nine articles were elected, both analyzed qualitatively, through methodological reading. Even though medicinal herb is known for its psychological use, recent studies show that cannabinoids (Cbs) are actively involved in peripheral systems such as the innate, adaptive immune response. The endocannabinoid system is responsible for regulating several aspects of skin homeostasis such as proliferation, release and differentiation of inflammatory mediators. Therefore, it was possible to observe through the research that Cannabis sativa and its system present in the organism, has large participations for the control of several dermatological and non-dermatological diseases, however, studies are still quite recent and scarce regarding the effect of the herb in the dermatological area, however, already has great advances on its effectiveness.
\end{abstract}

Keywords: Cannabis; Cannabinoids; Skin diseases.

\section{Resumen}

El referido artículo se trata de una revisión bibliográfica sistemática realizada en el período de 25 de octubre a 04 de noviembre 2021 a través de búsqueda electrónica en las bases de datos de Google Académico, Science Direct y PUBMED-NCBI-NIH para la obtención de datos más recientes, la pregunta guía de la investigación fue: ¿Cuál es la importancia de los cannabinoides para el tratamiento de fisiopatologías dermatológicas? Como criterio de elegibilidad se han seleccionado artículos de los últimos tres años referentes al cannabis, sus cannabinoides y el tratamiento de lesiones dermatológicas. Los descriptores utilizados fueron: Cannabis, SkinDiseases y Cannabinoidsverificados en los Descriptores en Ciencia de la Salud (DeCS) y en el Medical SubjectHeadings (MeSH). Se ha sucedido el cruce utilizando el operador booleano AND: "Cannabis" AND "Skindiseases" AND "Cannabinoids". Fueron elegidos 9 artículos, ambos analizados de forma cualitativa, a través de lectura metodológica. Aunque la hierba medicinal es conocida por su uso psicológico, estudios recientes muestran que los cannabinoides (CBs), están activamente involucrados en sistemas periféricos, como la respuesta inmune, innata y adaptativa. El sistema endocannabinoide es responsable y funcional de regular diversos aspectos de la homeostasia de la piel como proliferación, liberación y diferenciación de mediadores inflamatorios. Por lo tanto, fue posible observar a través de la investigación que el Cannabis sativa y su sistema presente en el organismo, tiene grandes participaciones para el control de diversas enfermedades dermatológicas y no dermatológicas, sin embargo, los estudios son todavía bastante recientes y escasos con respecto al efecto de la hierba en el área dermatológica, sin embargo, ya tiene grandes avances en su eficacia.

Palabras clave: Canabis; Cannabinoides; Enfermedades de la piel.

\section{Introdução}

Os canabinoides se referem às substâncias presentes na planta Cannabis sativa e aos receptores do sistema endocanabinóide presentes no organismo. A Cannabis sativa é uma espécie herbácea do gênero cannabaceae que vem sendo 
utilizada como um alimento, fonte de fibra, fármaco e medicamento, desde a antiguidade (Guida et al., 2019). A família Cannabis possui três espécies diferentes, sendo a mais habitual e rica em propriedades terapêuticas a da espécie Cannabis sativa, já a tipo Cannabis indica apresenta um teor mais baixo de tetrahidrocanabidiol (THC) que é a substância psicoativa da erva, também tem a Cannabis ruderalis, que não possui as substâncias psicoativas. O sistema endocanabinóide possui uma formação os quais envolvem receptores chamados de CB1 e CB2 que estão acoplados a proteína G, aspartato químico e ligantes endógenos também estão incluídos no sistema, sendo que o último citado é responsável por sintetizar e finalizar as atuações dos outros dois elementos. (Tescarollo, 2020).

A cannabis sativa possui diversas propriedades terapêuticas, contendo mais de 400 elementos e 60 são compostos por canabinóides, é essa espécie que é indicada para fins terapêuticos. O principal psicoativo da cannabis sativa é o Delta - 9-THC (tetrahidrocanabidiol) que possui uma ação complexa no cérebro (Pessoa, 2021). O D9-THC possui efeito anestésico e inflamatório, tendo seus efeitos comparados aos da morfina (Silva \& Freitas, 2021).

Dentre as propriedades terapêuticas, se destaca com significativas melhoras no tratamento de doenças neurodegenerativas ou psiquiátricas, a planta apresenta alivio nos casos de epilepsia, mal de Parkinson, Alzheimer, doenças inflamatórias e entre outras. Para a oncologia refere-se uma significativa melhora no controle da dor oncológica (Neto, 2020). No entanto, a utilização da Cannabis sativa na quimioterapia também está associada a eficácia em relação a náuseas e vômitos, pois, tais sintomas não são controlados com outros medicamentos (Pessoa, 2020).

Os canabinóides mostram uma abrangente capacidade terapêutica em doenças do sistema nervoso. Entretanto, estudos avançam em relação a planta e a sua espécie medicinal para com a área dermatológica, considerando que as doenças do sistema tegumentar apresentam um nível baixo de mortalidade, porém, quando relacionadas a prurido e dor é inegável que acarreta impacto na qualidade de vida e na autoestima do paciente, que pode vim a apresentar efeitos psicológicos de caráter negativos, além das modificações fibróticas que resultam eventualmente em cicatrizes definitivas (Freire, 2020). Os canabinoides tópicos são apresentados pela indústria como uma considerável possibilidade de tratamento para algumas doenças dermatológicas, o uso da Cannabis Sativa, está cada vez mais presente nos países europeus, Austrália e Canadá, onde os profissionais da medicina apresentam cada dia mais prescrições com produtos derivados da Cannabis (Viana et al., 2021).

A pele é o maior órgão do corpo humano e possuem funções relacionadas a regulação da temperatura, sensibilidade, barreira de proteção e controle de perda hídrica. É composta pela epiderme, derme e tecido subcutâneo, em casos de traumas e lesões, podem desenvolver o processo de cicatrização, compostos pela fase inflamatória, proliferativa e reepetelização (Lima, 2019). Dessa forma, considera-se que o sistema endocanabinóide possui uma relação com a homeostasia da pele, no que interfere o crescimento, diferenciação e até mesmo a sobrevivência das células, como respostas inflamatórias e imunes. Sendo assim, muitas patologias do sistema tegumentar podem estar relacionadas ás modificações deste sistema, como: cicatrização anormal, fibrose exuberante e esclerose sistêmica (Paiva, 2019). A cannabis Sativa também mostrou benefícios que podem ser utilizados como recursos terapêuticos para o tratamento da acne e conseguir combater a bactéria Propionibacterium acnes que pode se tornar resistente diante outros tratamentos (Cavalcante et al., 2021).

Dessa maneira, a falta de regulação do sistema endocanabinóide envolvendo o CB2, pode estar relacionado com o desenvolvimento de algumas doenças patológicas que impliquem uma fibrose exuberante da pele, processos de cicatrização anormal, esclerose sistêmica e também pode facilitar o desencadeamento de outras patologias cutâneas como a acne, dermatite alérgica, psoríase, dermatite seborreica e neoplasia da pele (Paiva, 2019).

O receptor CB1, presente no sistema endocanabinóide é o agente responsável pelos efeitos neurocomportamentais dos canabinóides e está situado nos terminais pré-sinápticos. Já o CB2 é o receptor presente no sistema imune, no entanto, também se expressa em neurônios. A proteína G está acoplada a esses receptores e é responsável por intermediar a transmissão do sinal entre CB1 e CB2 e também a níveis múltiplos, como enzimas e canais iônicos (Tescarollo, 2020). O CB2 está presente em 
diversas células, principalmente nas periféricas e imunes. Assim sendo, o CB2 está envolvido em diversas patologias. E os endocanabinóides são feitos a partir dos precursores lipídicos de membrana, quando há necessidade pode ser instigado a produzir estímulos fisiológicos ou patológicos. Logo, o receptor CB2 pode ser ativado "sob demanda", no caso, quando necessário (Lima, 2019).

Outro elemento é o $\beta$ - cariofileno, um sequiterpeno natural, ou seja, um tipo de terpeno, em outras palavras é uma substância natural produzida por vegetais, são um grupo de metabólicos secundários presentes em uma devasta variedade de família de plantas, uma dessas é a família Cannabis da espécie sativa. Dessa forma, o $\beta$ - cariofileno é um canabinóide antiinflamatório que tem um grande potencial de se ligar e atuar como agonistas no receptor CB2, posto isso, ocorre a ativação e inibição das vias de sinalização que promovem a expressão de citocinas pró-inflamatórias e a resposta imunitária TH1, diante disso, inibe a expressão de citocinas pró-inflamatórias e atua na redução da inflamação (Lima, 2019). Acredita-se que elementos com atividades agonistas nos receptores CB2 estão disponíveis e autorizados, representando um potencial terapêutico no caso da fibrose cutânea patológica (Paiva, 2019).

No entanto, a utilização da cannabis ainda é um tabu na sociedade, por ser associado ao passado, visto como uma marginalização e discriminação, desde os primórdios até os dias atuais (Neto, 2020). Mesmo com o avanço dos estudos sobre a planta e fins terapêuticos para diversas fisiopatologias, ainda há muito a ser superado na atual sociedade.

Dessa forma, o presente estudo visou buscar e analisar a revisão da literatura de forma sistemática, com a finalidade de compreender e expor os possíveis benefícios da cannabis sativa e o sistema endocanabinóide para o tratamento terapêutico de fisiopatologias dermatológicas, considerando que é uma abordagem recente, diante dos inúmeros estudos sobre os benefícios relacionados às doenças neurodegenerativas. Estudos como este se faz de tamanha relevância para contribuir com os avanços de pesquisas sobre a utilização da maconha medicinal (Cannabis sativa) no contexto dermatológico.

\section{Metodologia}

O referido artigo trata-se de uma revisão bibliográfica sistemática do tipo narrativa, realizada nos meses de outubro a novembro de 2021 através de pesquisa eletrônica nas bases de dados do Google Acadêmico, Science Direct e PUBMEDNCBI-NIH para obtenção de dados mais recentes, a questão norteadora da pesquisa foi: Qual a importância dos canabinoides para o tratamento de fisiopatologias dermatológicas?

Como critério de elegibilidade foi selecionado artigos dos últimos três anos referentes à Cannabis, seus canabinoides e o tratamento de lesões dermatológicas. Os descritores utilizados foram: Cannabis, Skin Diseases e Cannabinoids verificados nos Descritores em Ciência da Saúde (DeCS) e no Medical Subject Headings (MeSH). Sucedeu-se o cruzamento utilizando-se do operador booleano AND: "Cannabis" AND "Skin diseases" AND "Cannabinoids".

Os critérios de inclusão adotados foram: I) estudos que respondessem à questão norteadora sobre a importância dos canabinóides para o tratamento de fisiopatologias dermatológicas no geral, a partir da leitura do título e resumo; II) período de publicação entre os anos de 2019 a 2021; III) estar nos idiomas português, inglês ou espanhol. Os critérios de exclusão envolveram estudos duplicados e que respondessem a revisão integrativa, livros, cartas ao editor e artigos de nota prévia. Em seguida, foi selecionado o quantitativo de nove artigos para compor o corpus de análise de artigos elegíveis.

Foram eleitos nove artigos, ambos analisados de forma qualitativa, através de leitura metodológica. Os resultados foram apurados de diversas fontes, sumarizados e retratados no estudo. O número reduzido de estudos selecionados se deu pelos seguintes motivos: ao analisar a breve leitura do título ou resumos, foi visto a incompatibilidade do tema em questão, existem muitos estudos a respeito da Cannabis, no entanto, foi priorizado a Cannabis Sativa, erva medicinal e seus benefícios para fisiopatologias dermatológicas, como caráter de inclusão foram os estudos os quais abordavam quais fisiopatológicas 
dermatológicas e o efeito da Cannabis Sativa sobre essas, também foi considerado os tipos de estudos não inclusos na pesquisa, seguindo os critérios de exclusão, mencionados acima. Desse modo, foram possíveis os seguintes resultados: Google Acadêmico (2019 a 2021) - 3.740 (selecionados: 3); SCIENCE DIRECT - 277 (SELECIONADOS: 2); PUBMED - 55 (selecionados: 2); Portal BVS/MEDLINE: 16 (SELECIONADOS 2).

Quadro 1. Seleção dos artigos por bases de dados. Brasil, 2022.

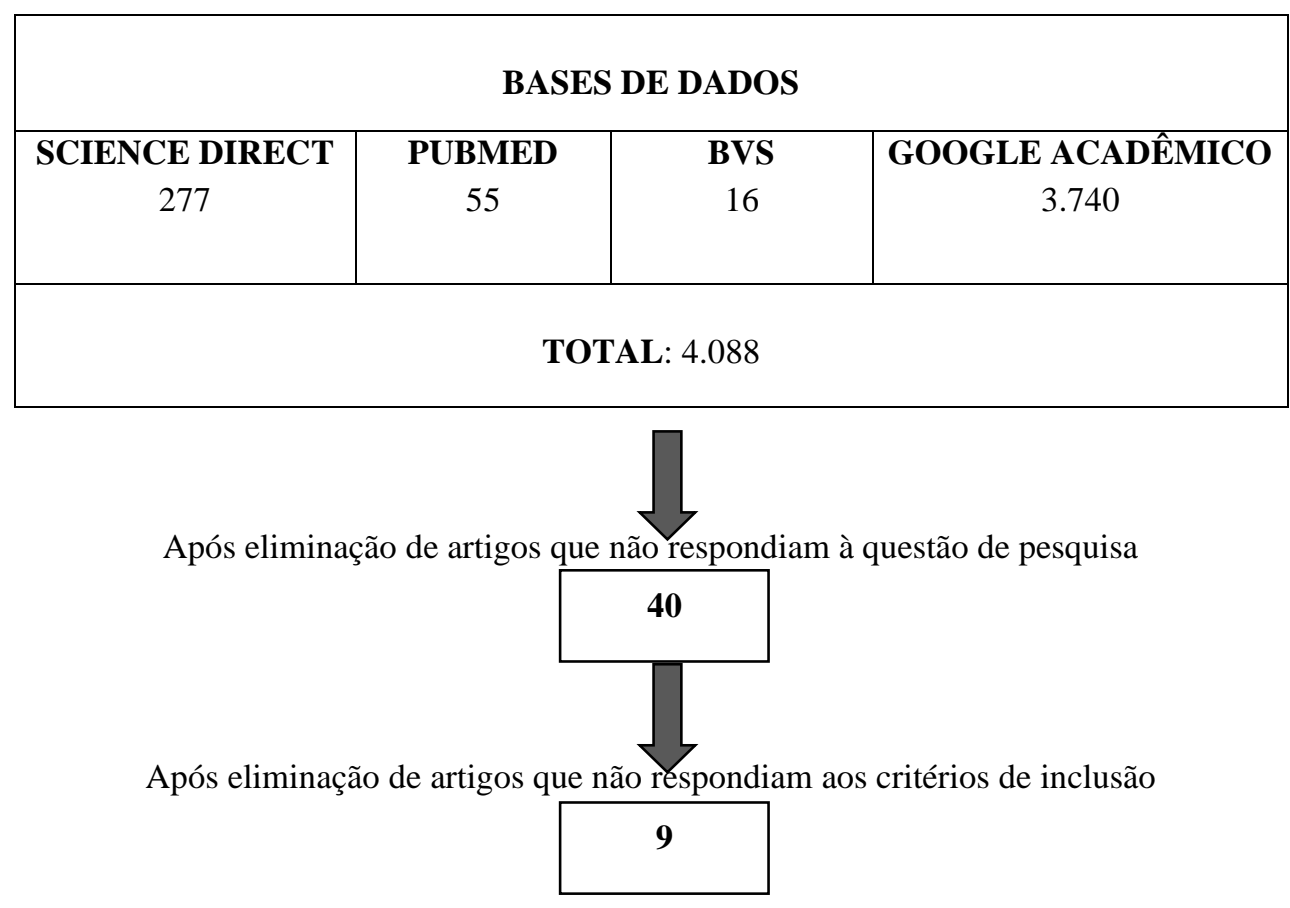

Fonte: Autores (2022).

\section{Resultados e Discussão}

Diante dos critérios de elegibilidade dos estudos, foi respeitado os critérios de inclusão e exclusão, sendo utilizados nove artigos para a composição do corpus de análise, selecionando estudos os quais respondiam à pergunta norteadora e que foram pulicados entre os anos de 2019 e 2021. As pesquisas mais atuais e que atingiram ao objetivo principal do estudo, foram agrupados no Quadro 1, contendo informações sobre autoria, ano de publicação, título e resultados. Objetivando uma coleta atualizada frente á importância dos canabinoides para o tratamento de fisiopatologias dermatológicas. 
Quadro 1. Corpus de análise de pesquisa. Brasil, 2021.

\begin{tabular}{|c|c|c|}
\hline $\begin{array}{c}\text { Autoria e ano de } \\
\text { publicação }\end{array}$ & Título do artigo & Resultados \\
\hline AVILA, C. et al., 2020. & $\begin{array}{l}\text { Cannabinoids for the treatment of } \\
\text { chronic pruritus: a review }\end{array}$ & $\begin{array}{l}\text { Quando se fala da utilização dos cannabinoides em prurido, tem } \\
\text { mostrado melhora no contexto de múltiplas doenças. Os } \\
\text { cannabinóides ópticos são de particular interesse em doença da pele } \\
\text { devido ao alto perfil de segurança e aplicação direta. }\end{array}$ \\
\hline $\begin{array}{l}\text { CINTOSUN, A. et al., } \\
2020 .\end{array}$ & $\begin{array}{l}\text { Mechanism of cannabinoids and } \\
\text { potential applicability to species } \\
\text { diseases }\end{array}$ & $\begin{array}{l}\text { Há um interesse crescente nos efeitos terapêuticos da } \\
\text { cannabinoides em dermatologia, visto que fornecem informações } \\
\text { de suporte sobre efeitos inibitórios dos cannabinoides na } \\
\text { inflamação, proliferação, fibrose, coceira e dor na pele. Esses } \\
\text { mecanismos fundamentam a fisiopatologia ou sintomas } \\
\text { desafiadores de uma série de condições dermatológica. }\end{array}$ \\
\hline $\begin{array}{l}\text { GUPTA, A. K. et al., } \\
2021 .\end{array}$ & $\begin{array}{l}\text { Cannabinoids for skin diseases } \\
\text { and hair regrowth }\end{array}$ & $\begin{array}{l}\text { Estudos clínicos preliminares em humano afirmam que os } \\
\text { cannabinóides pode melhorar eczema, acne, prurido cutâneo e } \\
\text { esclerose sistêmica. De acordo com a pesquisa, } 86 \% \text { dos } 531 \\
\text { dermatologistas foram positivos sobre os cannabinoides tópicos. }\end{array}$ \\
\hline $\begin{array}{l}\text { MILANO, R. et al., } \\
2019 .\end{array}$ & $\begin{array}{l}\text { Cannabinods: Potential role in } \\
\text { inflammatory and neoplastic } \\
\text { diseases. }\end{array}$ & $\begin{array}{l}\text { Dados recentes mostraram que o sistema endocanabinoide é } \\
\text { totalmente funcional na pele e é responsável pela manutenção de } \\
\text { muitos aspectos da homeostase cutânea, como proliferação, } \\
\text { diferenciação e liberação de mediadores inflamatórios. }\end{array}$ \\
\hline $\begin{array}{c}\text { SANGIOVANNI, E. et } \\
\text { al., } 2019 .\end{array}$ & $\begin{array}{l}\text { Cannabis sativa L. extractand } \\
\text { cannabidiol linhibit in vitro } \\
\text { mediators of skin inflammation } \\
\text { and wound injury }\end{array}$ & $\begin{array}{l}\text { O efeito do canabidiol e liberação de metaloproteinase } \\
\text { paralelamente ao efeito do extrato, tornando o canabidiol o } \\
\text { principal contribuinte ao efeito desejado. }\end{array}$ \\
\hline $\begin{array}{l}\text { SCHEAU, C. et al., } \\
2020 .\end{array}$ & $\begin{array}{l}\text { Cannabinoids in the } \\
\text { pathophysiology of skin } \\
\text { inflammation. }\end{array}$ & $\begin{array}{l}\text { Os canabinoides são vistos com interesse crescente como } \\
\text { medicamentos elegíveis no tratamento de doenças inflamatórias da } \\
\text { pele, com potenciais efeitos anticâncer, e a prontidão no } \\
\text { monitoramento dos efeitos e a facilidade de aplicação tópica podem } \\
\text { contribuir para o apoio crescente ao uso dessas substâncias. Apesar } \\
\text { dos resultados iniciais promissores, estudos humanos controlados } \\
\text { adicionais são necessários para estabelecer o papel definitivo desses } \\
\text { produtos na fisiopatologia da inflamação da pele e sua utilidade no } \\
\text { ambiente clínico. }\end{array}$ \\
\hline SHAO, K. et al., 2021. & Cannabis and the skin & $\begin{array}{l}\text { Dados encorajadores sugerem possíveis papéis terapêuticos para } \\
\text { cannabinóides m dermatologia como agentes anti-inflamatórios e } \\
\text { antiproliferativos. }\end{array}$ \\
\hline $\begin{array}{l}\text { SHERIFF, T. et al., } \\
2019 .\end{array}$ & $\begin{array}{l}\text { The potential role ofcannabinoids } \\
\text { in dermatology }\end{array}$ & $\begin{array}{l}\text { Cada vez mais os pacientes estão procurando um tratamento } \\
\text { natural, no estudo foi descoberto que } 94 \% \text { dos pacientes acreditam } \\
\text { que valem a pena a continuação da busca por novos estudos sobre o } \\
\text { papel e importância da cannabinoides na dermatologia. }\end{array}$ \\
\hline $\begin{array}{l}\text { TIJANI, A. O. et al., } \\
2021 .\end{array}$ & $\begin{array}{l}\text { Delivery of therapeutic } \\
\text { cannabinoids via the skin: current } \\
\text { status and future prospects }\end{array}$ & $\begin{array}{l}\text { O potencial de aplicação da cannabinóide detalhe o potencial por } \\
\text { via transdermica no tratamento da dor e inflamação, sendo eficaz } \\
\text { para melhorar os escores da dor. }\end{array}$ \\
\hline
\end{tabular}

Fonte: Autores.

A pele é representada como a via mais promissora para a entrega de cannabinoides, tanto topicamente quanto para indicações cutâneas. Sendo que, podem produzir diversos fármacos, principalmente os anticonvulsivantes, analgésicos, antiinflamatório e anti-emético neuroprotetor, tendo assim, uma ampla aplicação na gestão de uma série de condições, porém, é mais comum no tratamento da dor crônica (Tijano et al., 2021).

Contudo, mesmo que a espécie medicinal seja conhecida por seu uso psicológico, estudos recentes mostram que os canabinóides (CBs), estão ativamente envolvidos em sistemas periféricos, como a resposta imune, inata e adaptativa. O sistema endocanabinóide é responsável e funcional pela regulagem de diversos aspectos da homeostasia da pele como: proliferação, liberação e diferenciação de mediadores inflamatórios (Milano \& Friedman, 2018).

O sistema endocanabinóide (SECB) é composto por três componentes imprescindíveis 1- endocanabinóides, como 2araquidonoilglicerol ou (2-AG) e Naraquidonoiletanolamina ou (AEA); 2- receptores endocanabinóides e 3- as enzimas responsáveis pela síntese e degradação dos endocanabinóides. Esse sistema tem a possibilidade de se envolver na homeostasia 
da pele, desse modo, alguma irregularidade pode acarretar distúrbios da pele, como prurido, dermatite atópica, pigmentação da pele, acne, perda de cabelo ou crescimento. Esse elemento é sintetizado nos folículos pilosos, epiderme e glândulas sebáceas e liberados em uma quantidade correspondente a necessidade para manutenção da fisiologia normal da pele (Gupta \& Talukder, 2021). O SECB possui a finalidade de inibir a liberação de mediadores da inflamação presentes no processo de a cicatrização de feridas e processos inflamatórios que ocorrem na pele (Sangiovanni et al., 2019).

As doenças inflamatórias cutâneas mesmo que apresente uma baixa taxa de mortalidade como a dermatite alérgica de contato, acne e dermatomiosite, possuem um enorme impacto na qualidade de vida e na autoestima dos pacientes. Mesmo que tenha uma facilidade de diagnostico, aplicação de tratamento tópico e acompanhamento da evolução devido à localização superficial, representa um foco de investigação cada vez mais procurado (Scheau et al., 2020). O uso tópico de canabinóides para doenças de pele pode apresentar vantagens em decorrência da capacidade da sua ação local, mas, as suas duas principais vantagens são: que os danos colaterais respiratórios sejam bem menores do que por inalação e que ao contrário do uso oral, no uso tópico evita o metabolismo de primeira passagem. Ainda vale ressaltar que o Canabidiol (CBD) por ser um modulador alostérico negativo para receptor $\mathrm{CB} 1$, ele pode atuar e promover o crescimento do cabelo, considerando que quando estimulado o CB1 retarda o alongamento da haste capilar, reduzindo a proliferação de queratinócitos e estimulando a regressão prematura do folículo piloso (Gupta \& Talukder, 2021).

Sendo assim, os canabinóides têm efeitos antagonistas e agonistas no sistema endocanabinóide, que podem ativar ou inibir funções de proliferação de queratinócitos, produção de sebo, cabelo e inflamação. Dentre as fisiopatologias dermatológicas se encontram na maioria dos casos relações com os receptores CB1 e CB2, seja na ativação ou inibição. No caso da acne, a inibição do receptor CB2 fez a supressão da fabricação de lipídios basais, ou seja, os antagonistas do CB2 podem ser úteis para tratamento de alterações da pele como a não funcionalidade da glândula sebácea. Já na doença de pele fibrótica os receptores CB1 e CB2 podem modificar a resposta fibrótica em condições como esclerose sistêmica, morfeia e fibrose induzida por drogas, com a ativação do CB2 pode prevenir a fibrose cutânea e a infiltração de leucócitos teciduais. Na psoríase pode auxiliar na redução/inibição da proliferação de queratinócitos que é mediada pelo THC. (Sheriff, Lin, Dubin \& Khorasani, 2019).

Os canabinóides também podem ser importantes para o tratamento de câncer de pele melanoma e não melanoma, no entanto estudos precisam ser projetados a respeito. Entretanto, foi visto em um estudo com camundongos que a ativação de CB1 e CB2 está relacionada com a diminuição da angiogênese, proliferação, crescimento, metástase e um aumento do apoptose em tumores melanoma, porém, o CB2 apresenta mais propriedades de anti malignidade quando relacionado ao CB1 (Sheriff et al., 2019).

Em um estudo randomizado e cego, o creme do extrato de semente da cannabis a $3 \%$ reduziu significativamente a produção de sebo comparado com a utilização do creme base (controle) (Gupta \& Talukder, 2021).Os estudos trazem o desenvolvimento bem-sucedido da administração cutânea de produtos farmacêuticos tecnológicos para os cannabinoides (CBs), o que requer um conhecimento aprofundado da organização da pele que consiste em três camadas: a camada externa morta/córnea, epiderme viável e a derme (Tijano et al., 2021).

Alguns lugares no mundo legalizam a utilização da cannabis e dessa forma, tem aumentado o uso generalizado da erva e seus produtos associados, ambos clinicamente e recreativamente. Com isso os dados encorajadores sugerem possíveis papéis terapêuticos para canabinoides em dermatologia, como agentes anti-inflamatórios e antiproliferativos (Shao \& Kels, 2021).

A coceira é uma propriedade de uma diversidade de condições cutâneas e não dermatológicas, que inclui a dermatite alérgica de contato, insuficiência hepática ou renal, eczema atópico, distúrbios neurológicos e psiquiátricos, logo, a fisiopatologia do prurido é bastante complexa e envolve diferentes mediadores, como histamina, interleucina-31, leucotrieno 4 , 
substância B4 e substância P, em estudos com animais foi possível identificar a redução das respostas de coceira através dos mecanismos dependentes do receptor CB1, esse efeito antipruriginoso é oriundo do bloqueio ou antagonismo do CB1 (Cintosun et al., 2020). Além de auxiliar no tratamento de algumas fisiopatologias dermatológicas, a Cannabis sativa e seu sistema endocanabinóide também se fazem presente no alivio de sintomas com procedência de outras doenças, melhorando a qualidade de vida de pacientes não dermatológicos (Tijano et al., 2021).

O resultado antipruriginoso da ligação do receptor canabinóide no tecido nervoso periférico e central, pode estar atrelado as ações de diversos receptores, pois, experimentos em vários tipos de doenças de pele, apontam que a modulação individual dos canais de CB1, CB2 exibe uma redução significante do prurido, esse evento é bastante semelhante ao papel dos canabinóides na dor, ou seja, o aumento de ação do CB1 e CB2 resultam no alivio do prurido (Ávila et al., 2020).

Nos estudos, foram encontrados que o desenvolvimento de modelos animais para esses distúrbios tem auxiliado na investigação dos processos fisiopatológicos envolvidos, levando assim a um melhor entendimento do curso da doença e de possíveis mecanismos que podem reverte ou limitar o desenvolvimento de algumas patologias. Com o grande impacto na vida dos pacientes e no sistema de saúde têm motivado a pesquisa de novos tratamentos entre os quais, os CBs são vistos com interesse crescente devido aos seus resultados iniciais favoráveis e efeitos adversos limitados (Scheau et al., 2020).

\section{Conclusão}

Portanto, foi possível observar através da pesquisa que a Cannabis sativa e seu sistema presente no organismo, tem grandes participações para o controle de diversas doenças dermatológicas e não dermatológicas, contudo, estudos ainda são bastante recentes e escassos a respeito do efeito da erva na área dermatológica, no entanto, já se tem grandes avanços sobre sua eficácia. Além de controlar diversos sintomas e fisiopatologias que não são controladas com tanta eficácia com medicações existentes no mercado, melhora a qualidade de vida de pessoas com doenças crônicas de pele. Mas, é preciso superar o tabu e a descriminalização da planta para utilizá-la de forma mais ampla para pesquisas do seu uso terapêutico e medicinal, que já possui grandes comprovações no âmbito das Ciências em Saúde. Com isto, o trabalho teve o intuito em fortalecer as pesquisas atuais e, incentivar novas pesquisas acerca do tema para concretizar a eficácia da erva no tratamento das doenças dermatológicas.

\section{Referências}

Almeida, M. G., Silva, G. P., \& Lourenço, M. S. (2020). Os benefícios terapêuticos da cannabis sativa (CS) aos pacientes oncológicos: um olhar desmistificador - revisão de literatura. Medicina: https://sistema.atenaeditora.com.br/index.php/admin/api/artigoPDF/40423

Avila, C., Massick, S., Kaffenberg, B. H., Kwatra, S. G., \& Betchel, M. (2020). Cannabinoids for the treatment of chronic pruritus: A review. J Am Acad. Dermatol, 82 (5), 1205-1212. https://pubmed.ncbi.nlm.nih.gov/31987788/

Campos, L. P., Rua, M. A. V., \& Tescarollo, I. L. (2020). Uso de cannabis no tratamento de acne vulgar. Atena editora. Cap. 117-125. https://sistema.atenaeditora.com.br/index.php/admin/api/artigoPDF/43083.

Cavalcante, G., Rojas, M. Y., Maikot, S. C. V., Silva, A. P., \& Gomes, A. C. M. (2021). Avaliação antimicrobiana de um extrato de cannabis sativa frente ao crescimento da bactéria propionibacterium acnes. Revista Artigos, 25-1-9. https://acervomais.com.br/index.php/artigos/article/view/6001/3749

Cintosun, A., Corrales, I. L., \& Pope, E. (2020). Mechanisms of cannabinoids and potencial applicability to skin diseases. Clin. Drug Investig, 40 (4), $293-304$. https://pubmed.ncbi.nlm.nih.gov/32060787/

Grupta, A. K., \& Talukder, M. (2021). Cannabinoids for skin diseases and hairre growth. Wiley Online Library, 20 (9), 2703-2711. https://onlinelibrary.wiley.com/doi/abs/10.1111/jocd.14352\#: :text=Skin\%20disorders\%20and\%20hair\%20growth,hair\%20growth\%20via\%20multiple\%20m echanisms.

Guida, J. G., Navone, C. C. O., Pérez, M. D. R., González, A. L. C. \& Ramos, P. M. V. (2019). Cannabis medicinal como recurso terapéutico: estudo preliminar. Revista Med. Urug, 35(4). http://www.scielo.edu.uy/scielo.php?pid=S1688-03902019000400113\&script=sci_arttext\&tlng=pt

Lima, M. M. (2019). Ação do B-cariofileno sobre a viabilidade e proliferação de fribroblastos. Trabalho de conclusão de curso (Biotecnologia) - Universidade 
Research, Society and Development, v. 11, n. 2, e55411225961, 2022

(CC BY 4.0) | ISSN 2525-3409 | DOI: http://dx.doi.org/10.33448/rsd-v11i2.25961

Federal da Integração Latino-Americana. https://dspace.unila.edu.br/bitstream/handle/123456789/5536/TCC\%20II\%20-

$\%$ 20final.pdf?sequence=1\&isAllowed=y

Milano, R., \& Friedman, A. (2019). Cannabinoids: potecial role in inflammatory and neoplastic skin diseases. Am J Clin Dermatol, 20 (2), 167-180. https://pubmed.ncbi.nlm.nih.gov/30542832/

Paiva, A., \& Sá, I. C. (2019). O sistema endocanabinóides: receptores canabinóides e fibrose da pele. Mestrado Integrado em Medicina - Faculdade de medicina. https://repositorio-aberto.up.pt/bitstream/10216/120574/2/336353.pdf

Sangiovanni, E., Fumagalli, M., Paccheti, B., Piazza, S., Magnavacca, A., Khalilpour, S., Melzi, G., Martinelli, G., \& Dell’Agli, M. (2019). Cannabis sativa L. extract and cannabidiol inhibit in vitro mediators of skin inflammation and woun injury. Phytother Res., 33 (8), 2083-2093. https://pubmed.ncbi.nlm.nih.gov/31250491/

Scheau, C., Badarau, I. A., Mihai, L. G., Scheau, A. E., Costache, D. O., Constantin, C., Calina, D., Caruntu, C., Costache, R. S., \& Caruntu, A. (2020). Cannabinoids in the pathophysiology of skin inflammation. Molecules, 25 (3), 652. https://pubmed.ncbi.nlm.nih.gov/32033005/

Shao, K. Stewart, C., \& Kels, J. M. G. (2021). Cannabis and the skin. Clin. Dermatol, 39 (5), 784-795. https://pubmed.ncbi.nlm.nih.gov/34785006/\#: :text=In\%20vitro\%20and\%20in\%20vivo,agent\%20for\%20many\%20dermatologic\%20conditions

Sheriff, T., Lin, M. J., Dubin, D., \& Khorasani, H. (2019). The potential role of cannabinoids in dermatology. J. Dermatolog Treat, 31 (8), 839-845. https://pubmed.ncbi.nlm.nih.gov/31599175/\#: :text=Cannabinoids\%20have\%20demonstrated\%20anti\%2Dinflammatory,promising\%20alternative\%20to\%20t raditional\%20treatments

Silva, A. R. C., \& Lins, R. S. O. (2021). Acannabis sativa para fins terapêuticos: uma análise dos riscos e benefícios. Atena editora, cap. 6, 287-299. https://sistema.atenaeditora.com.br/index.php/admin/api/artigoPDF/44573.

Silva, M. L. O., \& Freitas, M. T. S. (2021). Analise toxicológica da cannabis sativa e seus benefícios terapêuticos. Brazilian Journal of Development, 7 (6), 63013-63023. https://www.researchgate.net/profile/Moises-

Souza, T. N., Abreu, Z. F., \& Vasconcelos, T. C. L. (2021). A utilização de canabinóides da fisiopatologia dermatológica - uma nova perspectiva de tratamento. Atenda editora. Cap. 9, 84-91. https://sistema.atenaeditora.com.br/index.php/admin/api/artigoPDF/52566.

Tijani, A. O., Thakur, D., Mishra, D., Frempong, D., Chukwuyere, U. I., \& Puri, A. (2021). Delivering therapeutic cannabinoids via skin: current state and future perspectives. J. Control Release, 10 (334), 427-451. https://pubmed.ncbi.nlm.nih.gov/33964365/

Tóth, K. F., Ádam, D., Biró. \& Oláh, A. (2019). Cannabinoid sing in the skin: therapeutic potential of the "C(ut)annabinoid" system. Molecules, 24 (5), 918. https://www.ncbi.nlm.nih.gov/pmc/articles/PMC6429381/

Viana, L. S., Silva, T. A., Antunes, V. M. S. \& Gonzaga, R. V. (2021). Efeito do óleo de Cannabidiol sobre a acne. Research, Society and Development, 10(14). https://rsdjournal.org/index.php/rsd/article/view/22075/19625 